\title{
Medición de la integración trabajo-familia en organizaciones
}

\section{RESUMEN}

El objetivo de esta investigación es desarrollar un instrumento para la medición de la Integración Trabajo-Familia (ITF) y su efecto organizacional. Es una investigación fundamentada en una revisión documental realizada anteriormente, a partir de la cual se elaboró el instrumento Indicadores ITF, aplicado a una muestra de 270 profesionales, empleados en diferentes empresas venezolanas. Se determinaron las dimensiones subyacentes a la ITF, con base en la percepción de los consultados, mediante el análisis de factores (método estadístico multivariado), siendo adecuado para el estudio con un KMO de 0,844. Este análisis arrojó como resultado cinco dimensiones para la medición de la ITF (Satisfacción ITF, Compromiso, Apoyo ITF, Doble Presencia y Efectos en la salud), con una varianza total explicada de $66 \%$ y a Cronbach superior a 0,644 para cada factor. Los resultados indican que la escala es fiable y válida para evaluar los componentes que determinan la ITF en las organizaciones.

Palabras clave: análisis de factores, diseño de escalas, integración trabajo-familia

MEASURING THE WORK-FAMILY INTEGRATION IN ORGANIZATIONS

\section{ABSTRACT}

The objective of this research is to develop an instrument for measuring Work-Family Integration (WFI) and its organizational effect. It is based on a literature review performed above, from which the instrument called WFI Indicators was drawn up. The instrument was applied to a sample of 270 professionals employees of different Venezuelan companies. WFI underlying dimensions were determined, based on the perception of respondents, by analyzing factors (multivariate statistical method) being suitable for the study with a KMO of 0,844 . This analysis yielded results in five dimensions for measuring the WFI (WFI satisfaction, commitment, support WFI, Double Presence and health effects), with a total explained variance of $66 \%$ and a Cronbach higher than 0.644 for each factor. The results show that the scale obtained is reliable and valid for assessing the components that determine the WFI in organizations.

Keywords: design scales, factor analysis, workfamily integration

\section{INTRODUCCIÓN}

Los estudios sobre la relación entre trabajo y familia, se han desarrollado desde la década de los setenta originando dos grandes vertientes. La primera comenzó a finales de los setenta, centrada en el análisis del conflicto experimentado por el sujeto, definido por Greenhaus y Beutell (1985) como una forma de conflicto de rol, en el que las presiones que resultan del trabajo y las responsabilidades familiares son mutuamente incompatibles en algún aspecto; la segunda, que convive con la anterior, pero comienza a cobrar fuerza a finales de mil novecientos ochenta, principios de los noventa, amplía el campo de estudio y así, una vez analizada la dimensión individual del conflicto, se orienta hacia el campo de las organizaciones, con el fin de conocer la experiencia corporativa y social, así como las soluciones propuestas tanto desde el ámbito público, como privado (Hernández, 2008). Es así como surgen términos como "conciliación", "equilibrio", "balance" entre trabajo y familia, los cuales están más relacionados con las políticas o acciones a realizar por los distintos entes, a fin de facilitar la disminución de los conflictos.

En esta investigación se asume una postura más positiva, en la relación entre trabajo y familia, sin dejar de lado la consideración de ambos enfoques, a través del uso del término Integración Trabajo-Familia (Integración T-F), entendiéndose por este como la respuesta afirmativa ante el conflicto, es decir al presentarse la tensión entre ambos roles, la persona consciente de la importancia de su desarrollo en estos dos ámbitos, aprovecha las facilidades que se les ofrece desde los diferentes actores (empresa, estado y otras instituciones), toma decisiones y actúa de manera que estas tensiones se minimicen, evitando impactar negativamente sobre el trabajo y la familia.

Independientemente del enfoque de las investigaciones, todas coinciden en que los efectos de la relación entre trabajo y familia, incide en tres aspectos: la organización, la familia y la persona misma. En lo que respecta al impacto sobre la empresa, diversos autores, coinciden en la dificultad que existe en medirlo de manera objetiva o directa, ya que la integración T-F, influye en indicadores que son multifactoriales, lo que hace más complejo establecer

* Ingeniero Industrial, Magíster en Administración de Empresas, mención Gerencia. Doctorando en Ingeniería, Área de Industrial, Universidad de Carabobo (UC). Profesor Asociado a Dedicación Exclusiva en la UC, Dpto. de Gerencia, Facultad de Ingeniería. E-mail: crisdalith@gmail.com, ccachutt@uc.edu.ve

** Ingeniero Industrial, Magíster en Administración de Empresas, mención Gerencia. Doctora en Ingeniería Industrial. Directora de la Dirección de Tecnología Avanzada de la Universidad de Carabobo. E-mail: prof.florangel@gmail.com, fortiz@uc.edu.ve 
la influencia específica de la integración T-F sobre ellos; por ejemplo: la intención de permanecer en la empresa depende de diversos elementos, como la retribución salarial, las expectativas de crecimiento profesional y no sólo de las facilidades para integrar la vida laboral y familiar.

Este estudio se enfoca especialmente en los efectos de la integración T-F, en el ámbito organizacional, por lo que el objetivo es desarrollar una escala que permita la medición de la Integración Trabajo-Familia (ITF) y su impacto en elementos concretos de tipo organizacional (Dicho instrumento se ha denominado Indicadores ITF); utilizando para esto el análisis de factores, como técnica estadística multivariante.

Como fundamento teórico se cuenta con una investigación previa realizada Cachutt y Ortíz (2015), dando como resultado una revisión exhaustiva de la relación entre trabajo y familia, así como de los efectos de esta en el rendimiento organizacional, esto sirvió de basamento para la elaboración del instrumento propuesto en el presente estudio. En la Tabla 1 se muestra una síntesis de los hallazgos de esta revisión.

\section{DISEÑO DEL INSTRUMENTO: INDICADORES ITF}

El diseño de la escala utilizada en este estudio, se desarrolló siguiendo las etapas propuestas por Lévy y Varela (2003), las cuales se muestran en la Figura 1.

En cuanto al objetivo del instrumento, este pretende medir el nivel de los Indicadores, relacionados con la Integración Trabajo-Familia (ITF) y su impacto en elementos de la organización. Para la elaboración de los ítems, en la revisión teórica no se encontraron evidencias de escalas específicas para medir el impacto de la relación entre trabajo y familia sobre el rendimiento organizacional, por lo que se generó una escala propia, partiendo de los distintos hallazgos teóricos. Dicho proceso se muestra con detalle en el trabajo realizado por Cachutt y Ortíz (2015).

Tabla 1. Efectos de la relación T-F, sobre la organización y su relación con la Integración T-F

\begin{tabular}{cllc}
\hline $\mathbf{N}^{\circ}$ & \multicolumn{1}{c}{ Efecto } & \multicolumn{1}{c}{ Fuentes } & $\begin{array}{c}\text { Relación con la } \\
\text { Integración T-F }\end{array}$ \\
\hline 1 & Ausentismo & $\begin{array}{l}\text { Kossek y Ozeki (1999), Baltes et al. (1999), citados por Eby et } \\
\text { al. (2005); Goff et al. (1990), Hammer et al.(2003), citados por } \\
\text { Hernández (2008), Chiavenato (2009), Chávez (2003) }\end{array}$ & Negativa \\
\hline 2 & $\begin{array}{l}\text { Retrasos en el } \\
\text { trabajo }\end{array}$ & Hammer et al. (2003), citado por Hernández (2008) & Negativa \\
\hline 3 & $\begin{array}{l}\text { Compromiso con } \\
\text { la organización }\end{array}$ & $\begin{array}{l}\text { Kossek y Ozeki (1999), citado por Eby et al. (2005), Kinnunen et } \\
\text { al. (2005), citado por Ugarteburu et al. (2008) }\end{array}$ & Positiva \\
\hline 4 & $\begin{array}{l}\text { Intención de } \\
\text { rotación }\end{array}$ & $\begin{array}{l}\text { Kossek y Ozeki (1999), citado por Eby et al. (2005); Boyar et al. } \\
\text { (2003), Greenhaus et al. (1997), Gradey y Cropazano (1999) y }\end{array}$ & Negativa \\
\hline 5 & Phaffer et al. (2001) citados por Hernández (2008) & Positiva \\
\hline
\end{tabular}

Fuente: Cachutt y Ortiz (2015)

Figura 1. Etapas para el diseño de escalas.

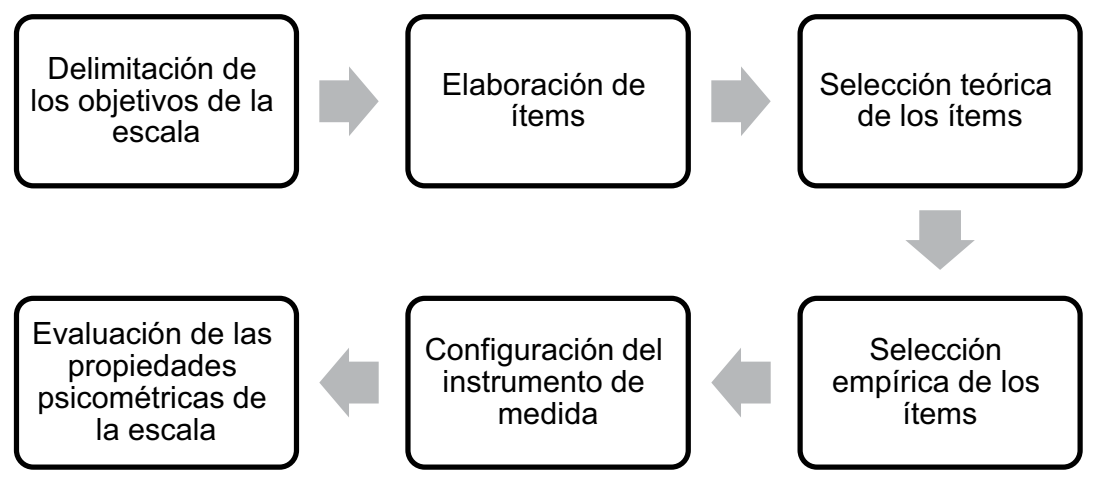

Fuente: Lévy y Varela (2003) 
El instrumento denominado Indicadores ITF, consta de 34 ítems, con formato de respuesta tipo Likert (del 1 al 5, como lo sugieren diversos autores entre ellos Lloret et al, 2014), gran parte de las variables provienen de adaptaciones de elementos de escalas existentes, como la de compromiso organizacional de Meyer y Allen (1991) en Chiang et al. (2010), factores psicosociales ISTAS21 (versión corta), Cuestionario del IESE Family Responsible Employer Index (IFREI), consultado en el trabajo de Cruz Rivas (2012). En esta fase que corresponde a la selección teórica de los ítems, se sometió a la revisión por parte de expertos, que sugirieron el ajuste de la redacción de varios elementos, así como la manera de presentar la escala, a fin de evitar inducir las respuestas de los encuestados.

El análisis empírico de los ítems se fundamenta en las respuestas obtenidas al administrar la escala a una muestra de personas Lévy y Varela (2003), una manera de comprobar esto fue dividiendo la muestra en dos partes de manera aleatoria y se realizó un análisis de las características de los ítems de manera independientes, se obtuvo características muy similares (consistencia interna, con Alfa de Cronbach entre 0.7 y 0.9 , y estructura interna), por lo que se puede afirmar que los resultados dependerán solo de la muestra, ya que la relevancia, representatividad y precisión de la redacción de los ítems conducen a los mismo resultados. Para la consistencia interna, se analizaron las contribuciones individuales de cada variable al coeficiente global, encontrándose algunas cuyas contribuciones no eran significativas y cuyas correlaciones con el resto de las variables eran muy poca (se descartaron aquellos ítems irrelevantes y ambiguos). Este análisis de consistencia y estructura interna de la escala permitió depurar la cantidad de variables a una cantidad tal que permitiera el uso adecuado del Análisis de Factores, usando el paquete estadístico SPSS 20. En total la escala quedó compuesta por 20 ítems.

En cuanto a la configuración del instrumento, se consideraron todas las observaciones dadas por los expertos en cuanto a la presentación de los ítems y las escalas, se elaboraron instrucciones claras acordes a las características de la muestra a consultar. También se presentaron ítems redactados de forma desfavorable, los cuales fueron luego codificados de manera inversa para el análisis estadístico, de igual manera se trató en la medida de lo posible, presentar los elementos de manera aleatoria para evitar inducir respuestas.

La comprobación de las propiedades psicométricas, constituye el último paso en la construcción de una escala; ésta será válida y fiable, en la medida que mida realmente el atributo que se pretende medir y además de manera precisa (Lévy y Varela, 2003).

La fiabilidad del instrumento Indicadores ITF, se determinó mediante el análisis de la consistencia interna que hace referencia al grado de relación existente entre los ítems que componen la escala. Con este propósito se usó el coeficiente Alfa de Cronbach, que es el indicador más utilizado para este fin; valores próximos a cero (0) indicarían una ausencia de consistencia interna y los cercanos a uno (1) consistencia interna entre los ítems de la escala (Lévy y Varela, 2003). Para la escala propuesta el Alfa de Cronbach para cada factor fue superior a 0,644 , esto indica una alta homogeneidad y equivalencia de respuesta a todas las variables a la vez y para todos los encuestados. La alta consistencia interna es un indicador suficientemente válido de que la escala usada mide la característica Integración Trabajo-Familia, lo que se interpreta como garantía de una alta fiabilidad del instrumento.

En cuanto a la validez de contenido, la escala Indicadores ITF considera las dimensiones importantes de la Integración Trabajo-Familia. Las treinta y cuatro (34) variables que integran la encuesta constituyen una adaptación, realizada tomando como referencia la literatura especializada en la relación entre trabajo y familia y sus efectos tanto en la persona, como en la organización, por lo que se puede considerar que la escala presenta validez de contenido.

\section{CARACTERÍSTICAS DE LA MUESTRA $Y$ PROCEDIMIENTO DE APLICACIÓN}

Dado que se busca medir la ITF y sus efectos en el ámbito organizacional, la población en estudio son profesionales (con estudios superiores, en cualquier carrera), con empleo fijo o dependiente. Para la aplicación de la escala se utilizó la herramienta Google Drive, la cual permitió hacer llegar vía correo electrónico el enlace, por medio del cual se accedía al instrumento, por lo que no hubo una escogencia intencional de los sujetos, ni de las empresas en las cuales laboran; estas podían ser tanto públicas, como privadas, de cualquier sector (manufacturero, servicios o comercialización).

De igual modo el instrumento se imprimió y se aplicó personalmente a diferentes grupos de estudiantes de postgrado, con las mismas características de profesión y empleo. En todos los casos, se dieron las instrucciones necesarias para responder adecuadamente el instrumento, así como garantizar un ambiente relajado y 
tranquilo. Los sujetos no recibieron ningún tipo de recompensa y su participación fue completamente voluntaria. La aplicación fue anónima, respetando así la confidencialidad de las respuestas dadas.

En cuanto al tamaño de la muestra, esta se ajustó considerando lo expresado por Lloret et al. (2014), que afirma que no solo es importante considerar el número de items por factor o la proporción de personas por elemento (que en este caso fue aproximadamente de 7 sujetos por ítems), sino que también hay que tomar en cuenta otros criterios como la comunalidad de los ítems. En este sentido, se evalúa la condición de la muestra, que para el caso de este estudio, es moderada, ya que se cumplen con las dos condiciones propuestas por Lloret et al (2014): las comunalidades deben estar entre .40 y .70 , y el número de variables por factor debe ser de 3-4 ítems, en estos casos estos autores sugieren que un tamaño de muestra, de al menos 200 sujetos es suficiente. En el caso específico de este estudio las comunalidades de las variables, son mayores a .4 en todos los casos (Ver Tabla 2).

Con base en esto, la muestra final quedó conformada por 270 individuos, lo cual supera las condiciones propuestas por los autores citados, lo que hace que la confiabilidad del estudio sea mayor. En la Tabla 3 , se presentan las características generales de dicha muestra. El procesamiento de los datos se realizó a través del paquete estadístico SPSS 20.
Tabla 2. Comunalidades de las variables

\begin{tabular}{cccc}
\hline VAR & Extracción & VAR & Extracción \\
\hline V21 &, 677 & V07 &, 591 \\
V23 &, 684 & V09 &, 733 \\
V18 &, 639 & V32 &, 762 \\
V17 &, 557 & V33 &, 655 \\
V28 &, 758 & V34 &, 798 \\
V29 &, 778 & V13 &, 526 \\
V30 &, 787 & V14 &, 598 \\
V31 &, 707 & V15 &, 560 \\
V06 &, 684 & V16 &, 418 \\
\hline
\end{tabular}

\section{DETERMINACIÓN DE LAS DIMENSIONES DE LA INTEGRACIÓN TRABAJO-FAMILIA}

Una vez evaluada la confiabilidad y validez del instrumento, se procede a determinar la contribución de los ítems en las dimensiones que conformarán los indicadores, que miden la ITF y sus efectos, a través del análisis factorial exploratorio.

Se demostró correlación entre las variables, ya que en la matriz de correlaciones se obtuvo coeficientes muy bajos (próximos a cero), además el estudio es significativo ya que el error de estimación cometido, en su mayoría no superó el nivel establecido, siendo

Tabla 3. Caracterización de la muestra

\begin{tabular}{|c|c|c|c|c|c|}
\hline \multirow[t]{2}{*}{ SEXo } & Femenino & Masculino & & & \\
\hline & $61 \%$ & $39 \%$ & & & \\
\hline \multirow[t]{2}{*}{ EDAD } & $\begin{array}{c}\text { Entre } 20 \text { y } 30 \\
\text { años }\end{array}$ & $\begin{array}{c}\text { Entre } 31 \text { y } 40 \\
\text { afios }\end{array}$ & $\begin{array}{c}\text { Entre } 41 \text { y } 50 \\
\text { afios }\end{array}$ & $\begin{array}{c}\text { Más de } 51 \\
\text { años }\end{array}$ & $\begin{array}{c}\text { PROMEDIO } \\
\text { EDAD }\end{array}$ \\
\hline & $40 \%$ & $34 \%$ & $17 \%$ & $9 \%$ & 35 años \\
\hline \multirow[t]{2}{*}{ ESTADO CIVIL } & Soltero & Casado & Divoreiado & $\begin{array}{c}\text { Con pareja } \\
\text { formal }\end{array}$ & Viudo \\
\hline & $45 \%$ & $40 \%$ & $11 \%$ & $24 \%$ & $4 \%$ \\
\hline \multirow[t]{2}{*}{ HiJos } & Tiene hijos & No tiene hijos & $\begin{array}{l}\text { Nro de } \\
\text { HiJos }\end{array}$ & $\begin{array}{c}\text { Entre } 1 \text { y } 2 \\
\text { hijos }\end{array}$ & $\begin{array}{c}\text { Más de } 2 \\
\text { hijos }\end{array}$ \\
\hline & $51 \%$ & $49 \%$ & & $82 \%$ & $18 \%$ \\
\hline \multirow{2}{*}{\multicolumn{3}{|c|}{ NIVEL DE EDUCACION }} & Doctorado & Maestria & $\begin{array}{l}\text { Educación } \\
\text { Superior }\end{array}$ \\
\hline & & & $5 \%$ & $25 \%$ & $70 \%$ \\
\hline \multirow{2}{*}{\multicolumn{3}{|c|}{ JORNADA DE TRABAJO DIARIA }} & $\begin{array}{c}\text { Más de } 8 \\
\text { horas }\end{array}$ & $\begin{array}{c}\text { Entre } 5 \text { y } 8 \\
\text { horas }\end{array}$ & $\begin{array}{c}\text { Menos de } 5 \\
\text { horas }\end{array}$ \\
\hline & & & $37 \%$ & $57 \%$ & $6 \%$ \\
\hline \multicolumn{4}{|c|}{ SECTOR DE LA EMPRESA DONDE LABORA } & Pública & Privada \\
\hline & & & & $54 \%$ & $46 \%$ \\
\hline
\end{tabular}


$p<0,05$. El determinante arrojó un valor cercano a cero, por lo que se puede afirmar que los ítems están linealmente relacionados y que a su vez es una matriz no singular.

La adecuación del modelo se verificó a través del índice KMO (Kaiser-Meyer-Olkin), el cual fue de 0,844 lo cual valida que el modelo de factores elegido es adecuado para explicar el comportamiento de los datos. A través del método de Extracción de Componentes Principales, se determinaron las variales correlacionadas agrupadas en factores, que explican la mayor parte de varianza total; de este análisis resultaron cinco (5) componentes, que según la regla de Kaiser explican el 66,19\% de la varianza total. Dicho valor es aceptable según
Hair et al. (2007), Lévy y Varela (2003), entre otros, que afirman que en estudios del comportamiento humano, donde la información es menos precisa, es normal considerar una solución que represente al menos $60 \%$ de la varianza total.

Para la interpretación de los datos, se realizó la rotación ortogonal de los componentes a través del método Varimax, se tomó como criterio considerar aquellas variables con valores superiores a 0,5. La definición de cada componente se realizó a partir de la interpretación de las variables que las conforman.

También se evaluó la consistencia interna de cada componente, arrojando valores mayores a 0,64. Todos estos resultados se resumen en la Tabla 4.

Tabla 4. Conformación y definición de las componentes (Indicadores ITF)

\begin{tabular}{|c|c|c|c|}
\hline \multicolumn{2}{|r|}{$\begin{array}{l}\text { COMPONENTE 1. SATISFACCIÓN ITF (SITF): Nivel de satisfacción personal, en relación a } \\
\text { la habilidad para integrar las responsabilidades laborales y familiares }\end{array}$} & \multirow[t]{2}{*}{ Peso * } & \multirow[t]{2}{*}{$\begin{array}{l}\text { Alfa de } \\
\text { Cronbach }\end{array}$} \\
\hline N. Var & Nombre de la Variable & & \\
\hline 28 & El modo en que usted divide el tiempo entre su trabajo y su vida personal o familiar & 0,838 & \\
\hline 30 & $\begin{array}{l}\text { Su habilidad para conciliar las necesidades de su trabajo, con las suyas personales } \\
\text { y las de su familia }\end{array}$ & 0,837 & 0,895 \\
\hline 29 & El modo en que su vida laboral, personal y familiar encajan y quedan integrados & 0,827 & \\
\hline 31 & La oportunidad que tiene de realizar bien su trabajo y también las obligaciones familiares & 0,774 & \\
\hline \multicolumn{4}{|c|}{$\begin{array}{l}\text { COMPONENTE 2. COMPROMISO (C): Nivel de apego a la organización y deseos de } \\
\text { permanecer en ella, debido a la valoración que recibe, tanto en lo personal, como familiar }\end{array}$} \\
\hline 23 & Me siento ligado (a) emocionalmente a mi organización & 0,810 & \multirow{4}{*}{0,791} \\
\hline 18 & Me siento orgulloso (a) de decir a otras personas que formo parte de esta organización & 0,736 & \\
\hline 21 & Esta empresa merece toda mi lealtad, pues se preocupa por mí y mi familia & 0,732 & \\
\hline 17 & Sería feliz si trabajara el resto de mi vida en esta empresa & 0,688 & \\
\hline
\end{tabular}

COMPONENTE 3. APOYO A LA ITF (AITF): Apoyo ofrecido por la organización, en cuanto a políticas de conciliación Trabajo-Familia, así como de formación y asesoría en ITF

\begin{tabular}{ll}
\hline 34 & $\begin{array}{l}\text { Seminarios, talleres o sesiones informativas, promovidas por la organización, sobre } \\
\text { la integración entre trabajo y familia }\end{array}$ \\
32 & $\begin{array}{l}\text { La asesoría profesional y/o personal que recibe por parte de la empresa, para integrar } \\
\text { trabajo y familia. }\end{array}$ \\
$33 \quad \begin{array}{l}\text { El apoyo que recibe por parte de la empresa en asuntos familiares, como flexibilidad } \\
\text { laboral, subsidio o guarderías para los hijos, etc. }\end{array}$ & 0,692 \\
\hline
\end{tabular}

COMPONENTE 4: DOBLE PRESENCIA (DP): Necesidad de atender simultáneamente las exigencias de trabajo y asuntos familiares

\begin{tabular}{lll}
\hline 9 & Hago gestiones familiares mientras estoy trabajando & 0,836 \\
$\mathbf{6}$ & Cuando estoy en el trabajo, pienso en asuntos familiares & 0,769 \\
7 & Hay momentos en los que necesitaría estar en la empresa y en casa a la vez & 0,700 \\
\hline
\end{tabular}

COMPONENTE 5. EFECTOS SOBRE LA SALUD (ES): Alteración de la salud física o emocional, que afectan el cumplimiento de los deberes en el trabajo y el hogar

\begin{tabular}{lll}
\hline 14 & Mis problemas emocionales me han limitado en el trabajo & 0,699 \\
13 & He tenido problemas de salud, que me han impedido realizar mi trabajo y labores en casa & 0,678 \\
15 & He sufrido problemas emocionales (como ansiedad, depresión o irritabilidad) & 0,668 \\
16 & Tengo dolores físicos & 0,625 \\
\hline
\end{tabular}

* Peso de la variable en la componente respectiva. Este valor es tomado de la matriz de componentes rotados (Método de extracción: Análisis de componentes principales. Método de rotación: Normalización Varimax con Kaiser. La rotación ha convergido en 6 iteraciones). 


\section{INDICADORES ASOCIADOS A LA INTEGRA- CIÓN TRABAJO-FAMILIA (INDICADORES ITF)}

Los indicadores ITF que resultaron de este análisis pueden clasificarse en dos categorías, la primera compuesta por aquellos que describen el tipo de ITF, bien sea por la satisfacción que manifiestan los sujetos, por la manera como integran ambas responsabilidades (Indicador 1, ver Tabla 5) o por el tipo de apoyo en cuanto a formación y adopción de Políticas de Conciliación Trabajo-Familia, dadas desde la empresa (Indicador 2).

La segunda categoría, está definida por los otros tres indicadores que se refieren a los efectos que conlleva la ITF: mayor o menor compromiso con la organización y deseo o no de permanecer en la empresa; doble presencia, que puede afectar el rendimiento laboral del trabajador, debido a la perdida de concentración, lo que pudiera también definirse como un tipo de ausentismo presencial (Cachutt y Ortíz, 2015) y finalmente los efectos sobre la salud, ya que es bien conocido que uno de los efectos de la falta de integración, es la tensión o estrés que puede desencadenar en múltiples manifestaciones psicosomáticas que de igual forma pueden repercutir en el rendimiento laboral del trabajador.

Tabla 5. Indicadores ITF

\begin{tabular}{cll}
\hline N. $^{\circ}$ & $\begin{array}{l}\text { Nombre del } \\
\text { Indicador }\end{array}$ & \multicolumn{1}{c}{ Tipo de Indicador } \\
\hline 1 & $\begin{array}{l}\text { Satisfacción } \\
\text { ITF }\end{array}$ & $\begin{array}{l}\text { Indicadores que caracterizan } \\
\text { el tipo de ITF vivida por el } \\
\text { sujeto }\end{array}$ \\
2 & Apoyo a la ITF & Indicadores referidos a la \\
3 & Compromiso & $\begin{array}{l}\text { medición de efectos de la ITF, } \\
\text { sobre elementos que pueden } \\
\text { afectar el rendimiento laboral } \\
\text { del trabajador }\end{array}$ \\
5 & $\begin{array}{l}\text { Efectos sobre } \\
\text { la salud }\end{array}$ & \\
\hline
\end{tabular}

\section{CONCLUSIONES}

La escala denominada Indicadores ITF, es una herramienta eficaz para analizar los elementos que definen la Integración Trabajo-Familia y sus efectos en personas con trabajo dependiente, ya que se demostró su validez y fiabilidad. Se partió de un instrumento compuesto por treinta y cuatro (34) ítems, resultando en una versión definitiva compuesta por dieciocho (18) variables, agrupadas en cinco factores, con una consistencia interna (alfa de Cronbach) superior a 0,64.

La conformación de la muestra permite afirmar que este instrumento puede ser aplicado a empleados profesionales, con relación de trabajo dependiente, de cualquier tipo de empresa sea pública o privada, en el sector industrial venezolano; sería interesante validar su aplicabilidad en sectores industriales de otros países.

Los indicadores ITF identificados a través de este estudio fueron: Satisfacción ITF, Apoyo a la ITF, Compromiso, Doble Presencia y Efectos sobre la Salud. Conocer y medir estos aspectos de la ITF, es útil para el establecimiento de estrategias y acciones a ejecutar desde la empresa, de manera que sus colaboradores, puedan integrar mejor las responsabilidades, tanto del ámbito laboral como familiar.

La determinación de estos Indicadores ITF, son un punto de partida para futuros estudios, ya que el análisis de factores asume que las componentes se comportan como dimensiones no correlacionadas, sin embargo resulta interesante medir si realmente entre ellas puede existir relación, ya que desde el punto de vista teórico éstas existen, por ejemplo se ha demostrado en investigaciones como las de Eby et al. (2005), Chinchilla et al. (2005), entre otros, que en la medida que se fomenta la adopción de Políticas de Conciliación T-F por parte de la empresa, el compromiso de los trabajadores aumenta, sin embargo, es interesante medir dichas relaciones y poder incluso determinar modelos cuantitativos al respecto. En este sentido, el análisis de ecuaciones estructurales, puede ser un método útil.

Así mismo se pueden estudiar los efectos causales de estos indicadores sobre aspectos concretos de rendimiento laboral, elementos que no han sido medidos, pero si corroborados desde la perspectiva teórica.

\section{REFERENCIAS BIBLIOGRÁFICAS}

[1] Cahutt, C. y Ortíz, F. (2015). Indicadores de rendimiento en la medición del impacto de la integración trabajo-familia. I Jornadas de Investigación Científica-Tecnológica Emergentes en Ingeniería Industrial. Valencia: Universidad de Carabobo.

[2] Chiang, M., Núñez, A., Martín, M. y Salazar, M. (2010). Compromiso del Trabajador hacia su Organización y la relación con el Clima Organizacional: Un Análisis de Género y Edad. 
Panorama Socioeconómico, Año $28, N{ }^{\circ} 40$, 92-103.

[3] Chinchilla, N., Poelmans, S., León, C. y Tarrés, J. (2005). Guía de Buenas Prácticas de la Empresa Flexible. Hacia la Conciliación de la Vida Laboral, Familiar y Personal. Madrid: Centro Internacional Trabajo y Familia.

[4] Cruz Rivas, D. (2012). Efectos de los entornos familiarmente responsables sobre la calidad de la Motivación de los Colaboradores. Pamplona: Servicio de Publicaciones de la Universidad de Navarra.

[5] Eby, L., Casper, W., Lockwood, A., Bordeaux, C. y Brinley, A. (2005). Work and family research in IO/OB: Content analysis and review of the literature (1980-2002). Journal of Vocational Behavior, 66, 124-197.

[6] Greenhaus, J. y Beutell, J. (1985). Source of conflict between work and family roles. Academy of Management Review, 10, 1, 76-88.
[7] Hair, J., Anderson, R., Tatham, R. y Black, W. (1999). Análisis Miltivariante, 5ta ed. Madrid: Prentice Hall Iberia.

[8] Hernández, A. (2008). Expectativas de la Vida Familiar y Laboral de una muestra de estudiantes Publicidad y Relaciones Públicas. Alicante: Universidad de Alicante .

[9] ISTAS21 (Instituto Sindical de Trabajo, Ambiente y Salud) (2003). Instrumento para la Prevención de Riesgos Psicosociales. Versión corta para empresas pequeñas y autoevaluación. Copenhague: ISTAS.

[10] Lévy, J. y Varela, J. (2003). Análisis Multivariable para las Ciencias Sociales. Madrid: Pearson Educación S.A.

[11] Lloret, S., Ferreres, A., Hernández, A. y Tomás, I. (2014). El análisis factorial exploratorio de los ítems: una guía práctica, revisada y actualizada. Anales de Psicología, 30 (3), pp. 1151-1169. 